\title{
Results of the whole-genome sequencing and annotation of the phenotype of bacteria of the genus Listeria
}

\author{
Marina Terekhova \\ St. Petersburg State University of \\ Information Technologies, Mechanics \\ and Optics, Saint-Petersburg, Russia \\ n.mim@mail.ru
}

\author{
Rogacheva Elizaveta \\ Saint-Petersburg Pasteur Institute, \\ Saint-Petersburg, Russia \\ elizvla@yandex.ru \\ Kraeva Lyudmila \\ Saint-Petersburg Pasteur Institute, \\ Saint-Petersburg, Russia \\ lykraeva@yandex.ru
}

\author{
Derevyanchenko Irina \\ Branch of the Federal State Health \\ Institution "Center for Hygiene and \\ Epidemiology in the City of St. \\ Petersburg", Saint-Petersburg, Russia \\ tamiirina110804@mail.ru
}

\begin{abstract}
The incidence of Listeriosis in recent years is constantly increasing. So, in highly developed countries it ranges from 0.3 to 1.5 , while in Russia - only $0.04 \%[1]$. Moreover, the indicators in Moscow and St. Petersburg exceed those in the whole country by 4 times, which is explained by the different quality of clinical and laboratory diagnostics. Moreover, mortality due to complications of listeriosis infection can reach $30 \%$ [2]. Therefore, the fact of contamination of food products with listeria is of great concern. In this case, the strains of the species $L$. monocytogenes, $L$. innocua, $L$. ivanovii, $L$. grayii, $L$. seeligeri, $L$. welshimeri are most often distinguished. And if earlier it was believed that representatives of the species $L$. monocytogenes possess the largest set of pathogenicity factors, then the possibilities of molecular and bioinformatics research methods provide a reliable picture with respect to other representatives of the genus Listeria, in particular $L$. innocua.
\end{abstract}

Keywords — Listeria, listeriosis, sequencing

Motivation and aim

Motivation

The incidence of Listeriosis in recent years is constantly increasing. Moreover, mortality due to complications of listeriosis infection can reach $30 \%$. Therefore, the fact of contamination of food products with Listeria is of great concern.

Aim

To study the pathogenic potential of strains of L. innocua.

\section{Methods}

The research material was 11 strains of bacteria of the genus Listeria isolated from food (ready-to-eat and semifinished products: 7 strains of $L$. monocytogenes and 3 strains of $L$. innocua), as well as 1 strain of $L$. monocytogenes from a patient with listeriosis. The classic bacteriological method, mass spectrometric, molecular (genome-wide sequencing), bioinformatic analysis of genetic data were used in the work.

\section{Results}

An analysis of the Listeria genomes showed that bacteria of the species L. innocua have in their genome a number of virulence genes characteristic of representatives of the species L. monocytogenes: clpC, which encodes the production of endopeptidase, prfA, which is responsible for the production of listeriolysin A, inlJ_1 and inlJ_2, which encode the production of protein internalin - an inducer of phagocytosis.

In addition, a plasmid with the ebrB gene characteristic of strains with multi-resistant properties was found in one strain of L. innocua isolated from a chicken semi-finished product. Moreover, the founded plasmid is derived from $L$. monocytogenes, and this species was also isolated from the same semi-finished product. All L. innocua strains had antibiotic resistance genes: from 20 to 30 for each strain. They included genes responsible for resistance to $\beta$-lactam antibiotics, fluoroquinolones, aminoglycosides, tetracyclines, macrolides, that is, to those groups of antibacterial drugs that are used in the treatment of listeriosis infection.

The results of phylogenetic studies showed a close relationship between the genomes of bacteria of the species $L$. monocytogenes and L. innocua. Therefore, the proximity of bacteria in food products, in places of their storage and transportation, can lead to faster transfer of genetic information from representatives of one species to another. At the same time, strains of L. innocua acquire pathogenicity and antibiotic resistance factors, which allows them to become hazardous to human health along with strains of the $L$. monocytogenes species. Therefore, further study of virulence markers in other representatives of the genus Listeria is necessary for therapeutic and anti-epidemic measures in foci of infection.

\section{REFERENCES}

[1] Alexandra Moura et al. Atypical Hemolytic Listeria innocua Isolates Are Virulent, albeit Less than Listeria monocytogenes. Infect Immun. 2019 Apr; 87(4): e00758-18.

[2] Glaser P., Frangeul L., Buchrieser C. et al. Comparative genomics of Listeria species // Science. 2016. Vol. 294. P. 849-852. 